\title{
Prediction of the Favorable Area for the Development of Fracture in Mud-Shale of Wufeng-Longmaxi Formation in Jiaoshiba Area, Sichuan Basin
}

\author{
Qianqian Wang, Lu Wang \\ School of Earth Sciences and Resources, China University of Geosciences, Beijing, China \\ Email: wangqianqian_cn@163.com \\ Received 12 February 2016; accepted 12 March 2016; published 15 March 2016 \\ Copyright (C 2016 by authors and Scientific Research Publishing Inc. \\ This work is licensed under the Creative Commons Attribution International License (CC BY). \\ http://creativecommons.org/licenses/by/4.0/

(c) (i) Open Access

\begin{abstract}
The main reservoir stratum of shale gas in the Jiaoshiba area, Sichuan basin is Wufeng-Longmaxi Formations. This paper calculates the tectonic difference stress values of JY1 well in different depths and numerically simulates the shale gas reservoir by elasto-plastic incremental method. The results of tectonic difference stress value suggest that the present tectonic stress values distribute strippedly in plane. The areas in favor of the development of fractures are stripped and mostly distributed in the northeast, middle and southwest of Jiaoshiba area, where the shale gas is concentrated.
\end{abstract}

\section{Keywords}

Cracks, Tectonic Stress Field, Numerical Simulation, Difference Stress

\section{Introduction}

The shale gas field in the Lower Silurian Longmaxi Formation in the Fuling, Sichuan Basin has been paid attention of the domestic and foreign scholars, which is the first shale gas field with one million cubic meters production. Some scholars suggest that the mud-shale reservoir is the main factor to affect the reservoir capacity and shale gas exploitation [1]-[4].

In recent years, many methods of cracks prediction are widely used, but finite element numerical simulation of the tectonic stress field is the most widely used [5]-[10]. This method was very popular in nearly 40 years, in most case, but the finite element numerical simulation was only elastic simulation, which is not consistent with a 
practical demonstration of plastic deformation. If the deformation of rocks is simulated accurately, the plastic simulation must be carried out. Although many people tried to modify the parameters of rheological equation to simulate the rheological characteristics of rock, the reliable rheological parameters are difficult to obtain. Therefore, it is impossible to the stress field simulation of "visco-elastic" [11] [12]. Wan Tianfeng Professor and Chen Mingyou Professor of China University of Geosciences established the elastic-plastic incremental method to solve this difficult problem, which was able to approximately simulate the plastic deformation of the rocks. This method is a near perfect finite element numerical simulation method with 2D structural stress field. The numerical simulation program and the simulation methods got continuous improvement and perfection during 30 years of application, and has succeeded in the study of structural fracture prediction in the oil-gas field and mineral exploitation [13]-[15].

There is a mud-shale reservoir enriched shale gas in the Lower Silurian Longmaxi Formation in the Jiaoshiba area, Fuling, Sichuan Basin. Therefore, we used elastic plastic incremental method to simulate the tectonic stress field in the Jiaoshiba area, predict the development of tectonic fractures and provide evidences for the favorable shale gas accumulation zones.

\section{Geological Background}

The Fuling shale gas field is located in Jiaoshiba, Fuling District of Chongqing City. Tectonically, this shale gas field is in the barrier type fold belt in the east of Sichuan Basin and the west of the Qiyueshan Fault, which is a special positive structure in the Wanzhou complex synclines (Figure 1).

Faulted anticline similar to a box shaped is the main tectonic in the Jiaoshiba area, which is different with narrow high steep anticlines along the both sides with NE or near NS trending. The Jiaoshiba structure is diamond shaped and boundaried by the two groups of reverse faults with NE and near NS trending respective, and separated by the fault-uplift, fault-depression with Qiyueshan Faults. The deformation of the Jiaoshiba structure is weak and the upper and lower tectonic layers are basically similar, which is like faulted anticline with box shaped, that is, relief top and small dip. Faults are not developed, while there are many faults in the steep flanks [4].

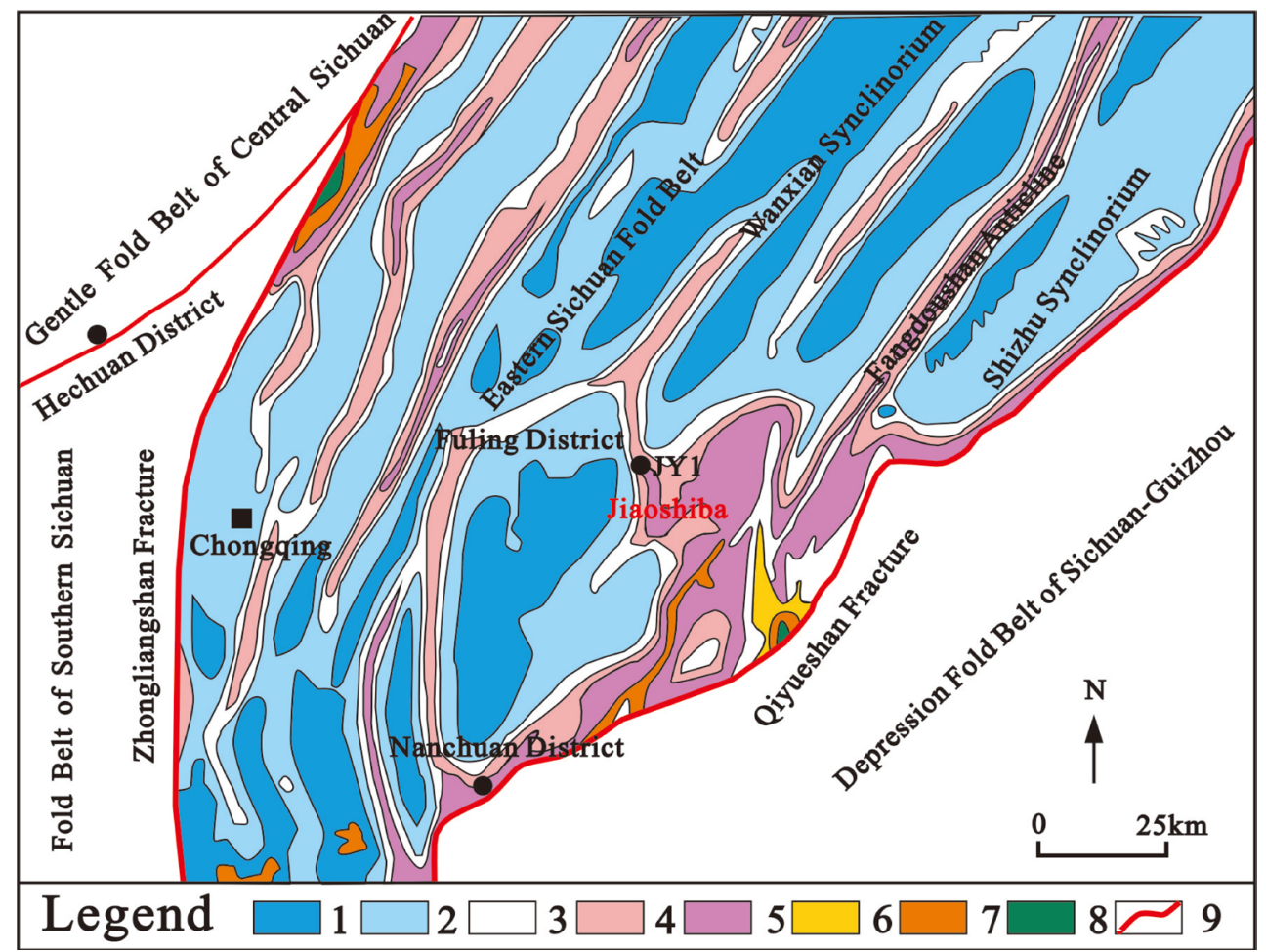

1. Upper Jurassic; 2. Middle Jurassic; 3. Lower Jurassic; 4. Triassic; 5. Permian; 6. Silurian; 7. Ordovician; 8. Cambrian; 9. Fracture 


\section{Analysis of Tectonic Stress Direction}

Because the present tectonic stress field is in the evolving, allocation and the formation process, those structure traces have not formed finally which can characterize the current tectonic stress field characteristics with a certain scale of permanent structure deformation. Therefore, the determination of the direction of modern tectonic stress is generally not by the geometry

At present, the methods are commonly used as following: analysis of the mechanism of natural earthquake, data of collapse in the engineering drilling, data of fault slip in the active faults and data of stress release in the borehole and so on.

Using natural earthquake focal mechanism solution method to determine the modern tectonic stress direction must be selected at the beginning of the earthquake or earthquake data, but can not use the aftershock data. After the formation of the earthquake, the aftershock, which usually only reflect a short-term, local stress state, can not be used as a parameter of the regional stress analysis. In recent decades, the domestic seismic network has been spread all over the country, and the method of solving the main stress direction with the focal mechanism is feasible and reliable.

Seismic source mechanism solution can be obtained by analyzing the seismic wave from the beginning to the record. In the process of seismic wave propagation, in each seismic station records with a seismic first arrival of the $\mathrm{P}$ wave (P-wave first motions of positive and negative (up and down) symbol and put them on the stereographic projection net, find out the classification of $\mathrm{P}$ wave first move plus and minus areas two faults (Figure 2).

Modern tectonic stress field maps of China have been compiled by the Crust Stress Research Institute of Chinese Academy of Sciences. The extrusion direction of Sichuan Basin is ca. SE120 in the modern tectonic stress field map of Sichuan Basin.

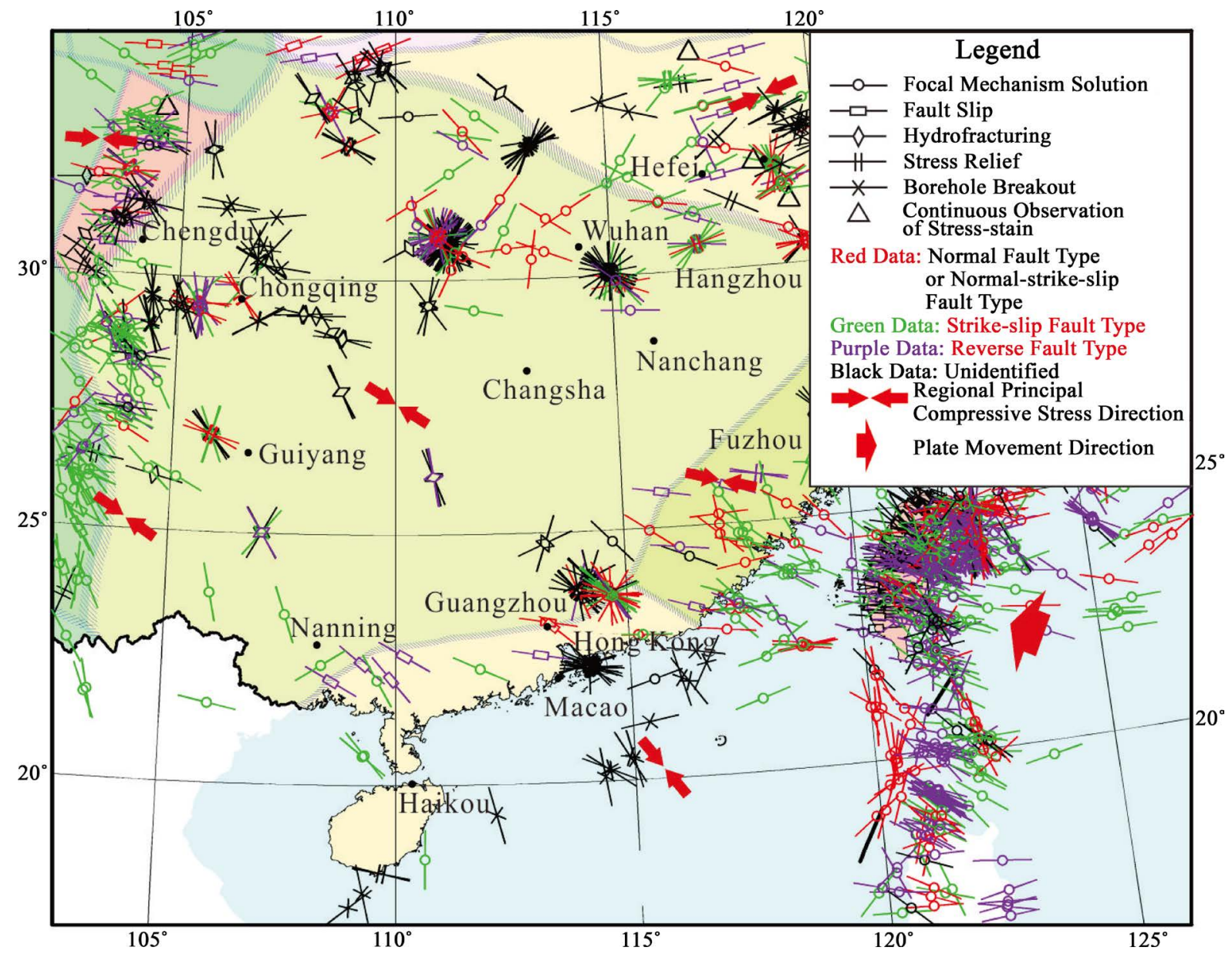

Figure 2. Modern tectonic stress field of Southern China. 
The modern tectonic stress field of Southern China suggests a direction of tectonic stress in a larger area. In order to the local direction of tectonic stress in the study area, we narrowed the scope of data collection and used the data of earthquake mechanism in the Jiaoshiba area, Sichuan Basin [16] [17] (Table 1) and the data of drilling collapse [18] (Table 2) to find the maximum compressive principal stress direction in the study area.

We calculated the the arithmetic average values of the 30 focal mechanism solutions and 15 drilling collapse data with result $122.98^{\circ}$, which is consistent with the direction (SE120 $)$ of the main pressure stress of modern tectonic stress field of Southern China. Therefore, SE $120^{\circ}$ has been choiced as the the direction of the main pressure stress of modern stress field simulation.

\section{Determination of Tectonic Stress by Hydraulic Fracturing Data}

We calculated the horizontal stress values in different depth (Table 3, Table 4), using the stress values and rupture pressure gradient in the fracturing report of JY1 well.

Table 1. Data of focal mechanism solution near the Jiaoshiba area.

\begin{tabular}{|c|c|c|c|c|c|}
\hline No. & Abscissa & Ordinate & Earthquake date & Place & Orientation $\left({ }^{\circ}\right)$ \\
\hline 1 & $18,347,157$ & $3,432,122.4$ & $2008 / 5 / 12$ & Wenchuan, Sichuan & 283 \\
\hline 2 & $18,387,483$ & $3,477,862.3$ & $2008 / 5 / 13$ & Pengzhou, Sichuan & 288 \\
\hline 3 & $18,327,963$ & $3,426,869.8$ & 2008/5/13 & Wenchuan, Sichuan & 289 \\
\hline 4 & $18,327,066$ & $3,471,255$ & $2008 / 5 / 16$ & Wenchuan, Sichuan & 276 \\
\hline 5 & $18,490,549$ & $3,569,626.7$ & 2008/5/18 & Pingwu, Sichuan & 316 \\
\hline 6 & $17,961,427$ & $3,338,864.3$ & $1989 / 4 / 25$ & Eastern Batang, Sichuan & 264 \\
\hline 7 & $17,974,099$ & $3,340,478.8$ & $1989 / 5 / 3$ & Eastern Batang, Sichuan & 271 \\
\hline 8 & $17,973,346$ & $3,344,969.2$ & $1989 / 5 / 3$ & Eastern Batang, Sichuan & 260 \\
\hline 9 & $17,967,872$ & $3,331,868.8$ & $1989 / 7 / 21$ & Eastern Batang, Sichuan & 269 \\
\hline 10 & $18,386,086$ & $3,222,078.2$ & $1994 / 12 / 30$ & Mabian, Sichuan & 272 \\
\hline 11 & $18,404,968$ & $3,486,892.9$ & $1958 / 2 / 8$ & Maowen-Beichuan, Sichuan & 296 \\
\hline 12 & $18,335,098$ & $3,393,482.3$ & $1970 / 2 / 24$ & WesternDayix, Sichuan & 305 \\
\hline 13 & $18,375,782$ & 3,168,972.4 & 1970/7/31 & Northeastern Leibo, Sichuan & 305 \\
\hline 14 & $18,380,956$ & 3,196,632.6 & $1971 / 8 / 17$ & Mabian, Sichuan & 316 \\
\hline 15 & $18,380,979$ & $3,198,849.5$ & $1971 / 8 / 17$ & Eastern Mabian, Sichuan & 354 \\
\hline 16 & $18,375,947$ & $3,184,490.7$ & $1971 / 8 / 17$ & Muchuan, Sichuan & 321 \\
\hline 17 & $18,365,243$ & $3,187,935.7$ & $1971 / 8 / 18$ & Eastern Mabian, Sichuan & 319 \\
\hline 18 & $18,375,900$ & $3,180,056.9$ & $1971 / 8 / 23$ & Eastern Mabian, Sichuan & 313 \\
\hline 19 & $18,348,285$ & $3,509,748.5$ & $1933 / 8 / 25$ & Northwestern Maowen, Sichuan & 274 \\
\hline 20 & $18,363,030$ & $3,165,789$ & $1971 / 3 / 11$ & Mabian, Sichuan & 297 \\
\hline 21 & $18,373,054$ & $3,187,847.7$ & $1971 / 8 / 23$ & Mabian, Sichuan & 308 \\
\hline 22 & $18,392,684$ & $3,198,735$ & $1973 / 6 / 29$ & Eastern Mabian, Sichuan & 265 \\
\hline 23 & $18,366,219$ & $3,187,924.4$ & $1971 / 9 / 4$ & Mabian, Sichuan & 286 \\
\hline 24 & $18,373,175$ & $3,198,932.4$ & $1973 / 6 / 29$ & Eastern Mabian, Sichuan & 301 \\
\hline 25 & $18,361,364$ & $3,190,198.7$ & $1971 / 11 / 5$ & Southern Mabian, Sichuan & 279 \\
\hline 26 & $18,392,787$ & $3,209,819.4$ & $1974 / 12 / 30$ & Mabian, Sichuan & 321 \\
\hline 27 & $18,323,276$ & $3,432,492.8$ & $1976 / 2 / 15$ & Wenchuan, Sichuan & 300 \\
\hline 28 & $18,335,607$ & $3,299,205.6$ & $1973 / 12 / 30$ & Southwestern Hongya, Sichuan & 284 \\
\hline 29 & $17,977,516$ & $3,310,252.1$ & $1960 / 5 / 3$ & Southeastern Batang, Sichuan & 283 \\
\hline 30 & $18,118,645$ & $2,892,441.3$ & $1979 / 5 / 15$ & Panxi, Sichuan & 277 \\
\hline
\end{tabular}


Table 2. Data of drilling collapse near the Jiaoshiba area.

\begin{tabular}{cccccc}
\hline No. & Abscissa & Ordinate & Depth & Place & Position $\left(^{\circ}\right)$ \\
\hline 1 & $18,709,564$ & $3,455,503.4$ & 4441 & Guan 8 & 264 \\
2 & $18,432,618$ & $3,472,373.3$ & 1985 & Chuanxi 106 & 280 \\
3 & $18,605,965$ & $3,480,788.6$ & 937 & Chuanbei 57 & 280 \\
4 & $18,597,714$ & $3,468,399$ & 2812 & Chuanbei 58 & 273 \\
5 & $18,381,695$ & $3,436,672$ & 3405 & Chuanxi 95 & 286 \\
6 & $18,436,653$ & $3,212,487.4$ & 2700 & Chuan 1 & 345 \\
7 & $18,437,634$ & $3,213,701.3$ & Chuan 2 & 335 \\
8 & $18,438,599$ & $3,212,033.5$ & 0 & Chuan 3 & 345 \\
9 & $18,440,504$ & $3,277,977$ & Chuan 4 & 355 \\
10 & $18,441,462$ & $3,275,976.7$ & Chuan 6 & 340 \\
11 & $18,507,442$ & $3,259,422$ & 2950 & Chuan 7 & 340 \\
12 & $18,520,469$ & $3,244,363.6$ & 3025 & Chuan 8 & 340 \\
13 & $18,683,350$ & $3,393,781.5$ & 2575 & Chuan 11 & 330 \\
\hline
\end{tabular}

Table 3. Calculation results of the fracturing report of JY1 well.

\begin{tabular}{cccccc}
\hline \multirow{2}{*}{ No. } & \multirow{2}{*}{ Layer } & Depth $(\mathrm{m})$ & & \multicolumn{3}{c}{ Stress $(\mathrm{MPa} \backslash \mathrm{MPa} / 100 \mathrm{~m})$} \\
\cline { 4 - 6 } & & & Overburden pressure & Maximum stress & Minimum stress \\
\hline JY1 & Longmaxi & 2380.70 & $58.62 \backslash 2.46$ & $63.50 \backslash 2.67$ & $47.39 \backslash 1.99$ \\
\hline
\end{tabular}

Table 4. Calculation results of the fracturing report of JY1 well.

\begin{tabular}{cccc}
\hline Depth $(\mathrm{m})$ & $\sigma_{1}(\mathrm{MPa})$ & $\sigma_{3}(\mathrm{MPa})$ & $\Delta \sigma(\mathrm{MPa})$ \\
1800 & 48.041 & 35.848 & 12.193 \\
2400 & 64.034 & 47.788 & 16.246 \\
3000 & 80.054 & 59.728 & 20.326 \\
3600 & 96.074 & 71.668 & 24.406 \\
4200 & 112.094 & 83.608 & 28.486 \\
4800 & 128.114 & 95.548 & 32.566 \\
\hline
\end{tabular}

\section{Establishment of Geological Model and Determination of Physical Property Parameters}

\subsection{Types of Deformation Medium}

Young's modulus and Poisson's ratio are the necessary rock mechanical parameters in the numerical simulation of tectonic stress field by the elasto-plastic incremental method. The response for stress is different for different kinds of deformation medium. The deformation medium in the study area can be divided into two categories by the continuity: non continuous deformation medium and continuous medium deformation.

\subsubsection{Non Continuous Deformation Medium}

The fault is a non continuous medium. Stress releases in the faults and the difference stress values decrease during the numerical simulation of tectonic stress field by the elasto-plastic incremental method. Therefore, the scale of faults plays an important role in the current tectonic stress field. Then, the fault should be acted as a kind of deformation medium. For the fault as a non continuous deformation medium, the key point is in the time-depth conversion of seismic data. We got the depth sections from height $-1800 \mathrm{~m}$ to height $-4800 \mathrm{~m}$, 
which include the fault tomographic point cutting this layer. In depth sections corresponding to the height-1800m however, Endo, these depth sections including wear fault tomographic point to this layer. These depth dections containing fault information are entered into DFDraw software. After coordinate correction of the depth sections and depiction of tomographic projection point, we got the distribution maps of faults corresponding to present simulation layers of tectonic stress (Figure 3). In the study area, the fault distance of layers is smaller and steep with different extension length. Meanwhile, the development characteristics of the faults are similar. The faults distribute along NE-SW. In the stress field simulation, the stress is affected by these faults, which is acted as one kind of media. So, we identified one kind of non continuous deformation media in the study area.

\subsubsection{Continuous Deformation Medium}

The continuous deformation medium would influenced by stratigraphic age and sedimentary environment. The sedimentary environment in the Jiaoshiba area, Sichuan Basin is relatively single, that is, shallow shelf facies. So, three kinds of continuous deformation mediums have been divided in the Longmaxi-Wufeng Formation, the overlaying of Longmaxi-Wufeng Formation and Ordovician limestone underlaying the Longmaxi-Wufeng Formation (Figure 4).

\subsection{Elastic Parameters of Tectonic Stress Field}

The elastic modulus and Poisson's ratio are the main parameters reflecting the characteristics of model material, which can be accurately determined in the testing machine of rock mechanics. Because these parameters would affect the simulation of plastic deformation process greatly during the mathematical simulations by this software, we must carefully select them. Generally, the measured data should be used as the first incremental elastic modulus and Poisson's ratio, because these data are tested under the condition of elastic deformation. However, the elastic modulus would significantly decreas during the second, three and four incremental stage, then we can simulate the process of plastic deformation. But the Poisson's ratio is just the opposite. Then, we should properly increase the Poisson's ratio In order to simulate the plastic deformation. The maximum value of the Poisson's ratio is 0.5 , therefore Poisson's ratio should not more than 0.45 even the presence of fault gouge or cataclasite.

The young's modulus and Poisson's ratio can be gotten from the shear wave logging data in the JY1 well of the study area. According to the small changes of the young's modulus and Poisson's ratio in different depths of JY1 well, average values of the young's modulus and Poisson's ratio in different depths would be taken as the simulation parameters (Table 5).

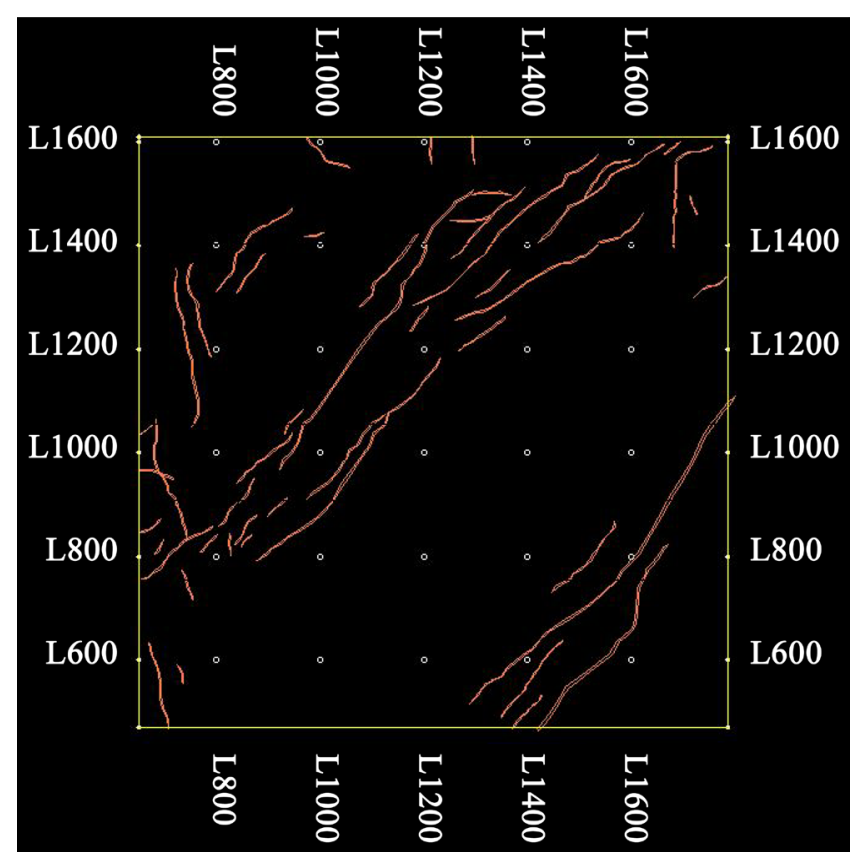

Figure 3. Horizontal sections of faults in the Jiaoshiba area. 

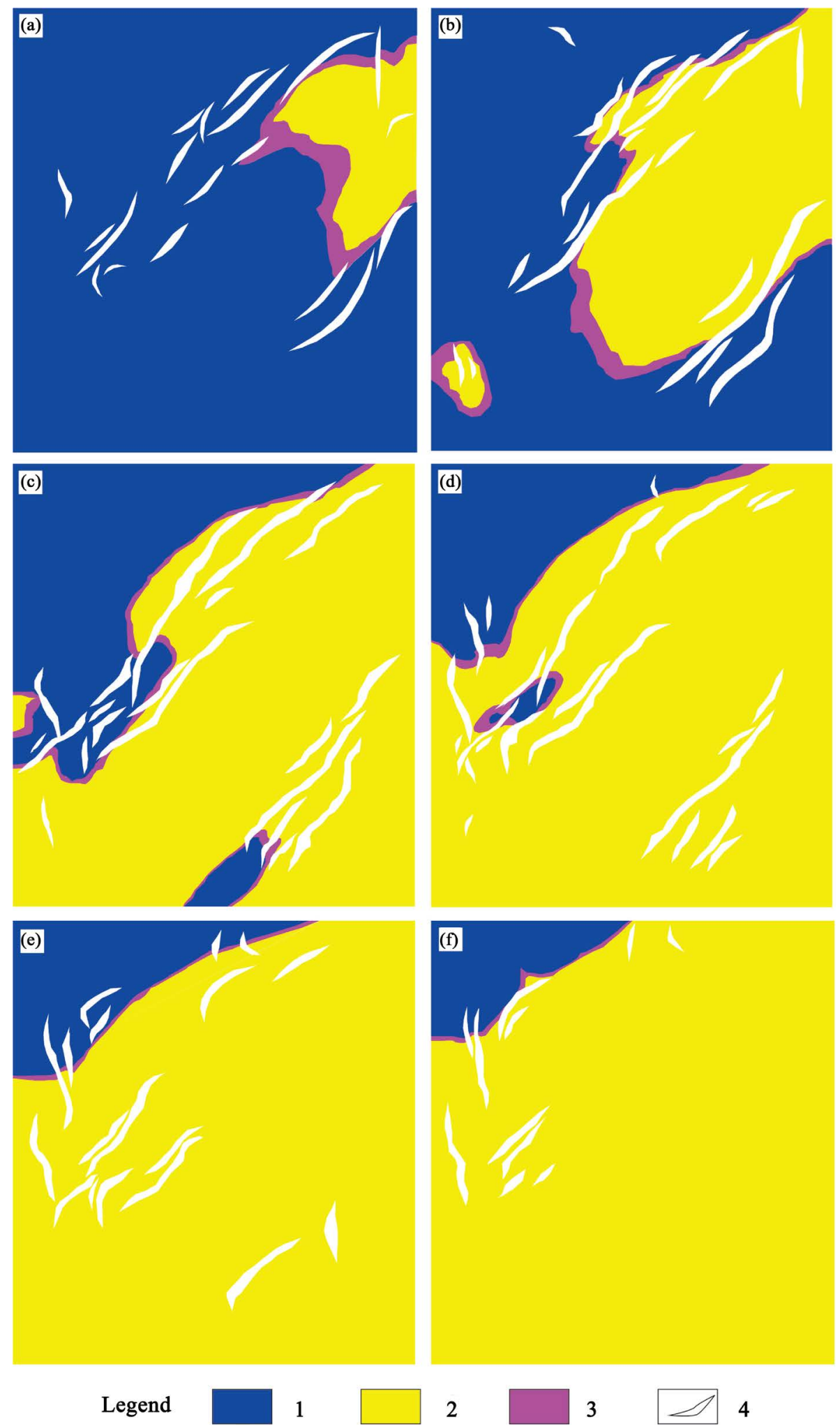

1: overlaying of the Wumafeng-Wufeng Fromation; 2: underlaying of the Wumafeng-Wufeng Fromation; 3: the Wumafeng-Wufeng Fromation; 4: Faults

Figure 4. Types of medium in different heights. (a) height-1800 m; (b) height-2400 m; (c) height—3000 m; (d) height—3600 m; (e) height-4200 m; (f) height—4800 m. 


\section{Numerical Simulation of Regional Stress Field}

\subsection{Simulation of Tectonic Stress Field by Elastic Plastic Incremental Method}

Elastic plastic incremental method simulates the plastic deformation process of the geological body and tectonic stress field finite element numerically by increment method. After nearly 30 years of exploration and repeated trial, Chen Mingyou Professor and Wan Tianfeng Professor of China University of Geosciences established the elastic-plastic incremental method to finite element numerical simulate two-dimensional structure stress field.

The study of rupture deformation of rocks suggests that there are two deformation stages from the deformation to rupture of rocks, i.e. elastic deformation stage and plastic deformation stage. In the elastic deformation stage, stress is proportional to the deformation, which shows a straight line with a steeper slope in the stress \& deformation map (Figure 5). During this stage, if the stress is removed, the rock will be immediately restored. With the continuation of the deformation process, the slope of the curve decrease in the stress \& deformation map. Then if stress is removed, the rock will not be restored. This is the stage of plastic deformation [19].

The elastic-plastic incremental method is characteristiced by the separation of the stress-deformation relationship in the plastic stage as many segments of linear, which are acted as a seires of elastic deformation process. This method can simulate the process of the rock deformation accurately. However, the majority of current mathematical simulation of stress field is only limited to simulate the elastic deformation. In fact, structural trace would not be produced in the elastic deformation. The rock will be restored with the removal of the stress.

Table 5. Physical property parameters of JY1 well.

\begin{tabular}{cccccc}
\hline Depth (m) & Property parameters & Above Longmaxi-Wufeng & Longmaxi-Wufeng & Ordovician limestone & Faults \\
\hline \multirow{2}{*}{1800} & Young's modulus (MPa) & 4.04 & 3.36 & 3.534 & 2.69333 \\
& Poisson ratio & 0.274 & 0.314 & 0.274 & 0.411 \\
\multirow{2}{*}{2400} & Young's modulus (MPa) & 4.54 & 3.86 & 3.831 & 3.02667 \\
& Poisson ratio & 0.269 & 0.309 & 0.2265 & 0.4035 \\
\multirow{2}{*}{3000} & Young's modulus (MPa) & 5.04 & 4.36 & 4.331 & 3.36 \\
& Poisson ratio & 0.264 & 0.304 & 0.2215 & 0.396 \\
\multirow{2}{*}{3600} & Young's modulus (MPa) & 5.54 & 4.86 & 4.831 & 3.69333 \\
& Poisson ratio & 0.259 & 0.299 & 0.2165 & 0.3885 \\
\multirow{2}{*}{4200} & Young's modulus (MPa) & 6.04 & 5.36 & 5.331 & 4.02667 \\
& Poisson ratio & 0.254 & 0.294 & 0.2115 & 0.381 \\
\multirow{2}{*}{4800} & Young's modulus (MPa) & 6.04 & 5.36 & 5.331 & 4.02667 \\
& Poisson ratio & 0.249 & 0.289 & 0.2065 & 0.3735 \\
\hline
\end{tabular}

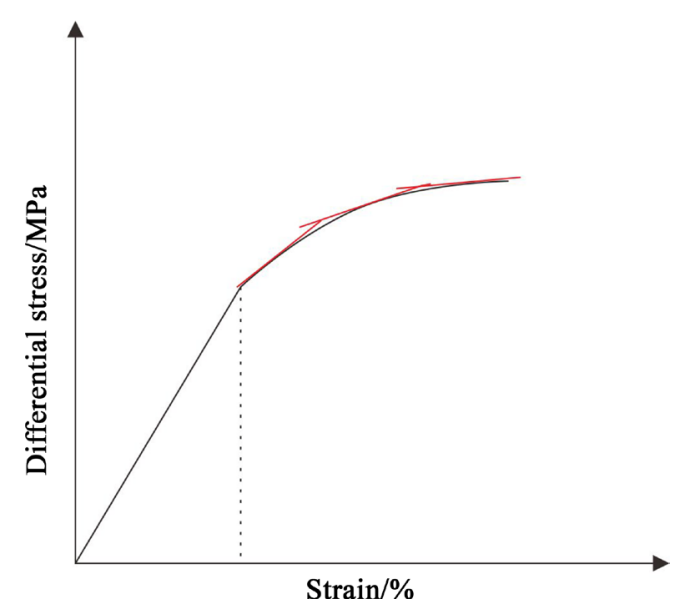

Figure 5. Schematic diagram of elastic plastic incremental method. 
External load force is applied around the designed geological model in order to produce the inner stress in the geological body, which is the key point of the numerical simulation of finite element structural stress field.

Generally, external load force should be evenly distributed around the model, in the best, evenly affect on the boundary line. However, when there are too many units with large grid density, it is convenient to apply a force on the node of the boundary of model and the accuracy is similar.

Generally, during the design of external load force on the boundary, it should be choised that the measured structural stress value with the same magnitude. The designed model should be approved when the direction of measured structural stress and the data of simulation are basically consistent in the model. However, it is difficult to achieve this goal unless repeated trial.

\subsection{Establishment of Mathematical Model and Prediction of Structural Fracture Zone}

\subsubsection{Establishment of Mathematical Model}

There are six numerical simulation layers in the study area $\left(400 \mathrm{~km}^{2}\right)$, which are $-1800 \mathrm{~m},-2400 \mathrm{~m},-3000 \mathrm{~m}$, $-3600 \mathrm{~m},-4200 \mathrm{~m}$ and $-4800 \mathrm{~m}$ isochronic lithology slices. We carried out the division of triangle units by four kinds of deformation media with different ages and different lithology. There are 15,546 of nodes, 354 of stress point of boundary, 2 constraint points of displacement and 3 times of increment.

\subsubsection{Basic Characteristics of Tectonic Stress Field}

Tectonic fractures form in the process of tectonic stress concentration and release. The fractures are not only depent on the size of tectonic stress, but also the release rhythm.

Some area is easy to the development of fractures where the stress gradient is relatively larger with the same stress value range [20]. Therefore, the structural stress values and changing gradient should be consided in prediction and research of the structure fracture. The study of tectonic cracks in oil-gas field suggests that, generally, the fracture zone in favor of the migration and accumulation of oil and gas are located in the area with middle difference stress values. During the process of plastic deformation, the biggest difference stress values, the smallest structural displacement. When the tectonic stress has not been released, the stress concentrated area is still in the elastic deformation stage and is not easy to the formation of cracks. However, the smallest difference stress values, the biggest structural displacement. When the tectonic stress has been released, the fractures and faults would develp perfectly. This is opposite with the elastic situation of the prediction of the fracture developed zone, but the oil-gas would leak if there are too many fractures. Therefore, the area with smaller difference stress values will be chosen but the area with smallest difference stress values in the fracture prediction of oil-gas field. In the Jiaoshiba area, the box shaped anticline is wide and flat, and several associated faults are mostly reverse faults with weak tectonic stress. Therefore, the area with smaller or smallest difference stress values has more tectonic fractures (Figure 6).

The area with low stress value (blue) is the stress releasing area. In the difference stress contour map (Figure 6(a), $-1800 \mathrm{~m}$ ), the difference stress values are lower in the northeast and southwest area than these of other regions. The lowest difference stress values are mainly concentrated in the JY1 wells in the northwest part of the study area, which is a narrow ends and wide middle area with NNE trending. There are two stress release centers in the northeast, east and southwest parts. Among them, the northeast stress relieve center has large gradient with most developed fractures. However, the southwestern and eastern stress release centers (blue area) have small gradient with developed tectonic fractures.

The area with low stress value (blue) is the stress releasing area. In the difference stress contour map (Figure 6(b), $-2400 \mathrm{~m}$ ), the difference stress values are lower in the northeast, center, southeast and southwest area than these of other regions. The lowest difference stress values are mainly concentrated in the JY1 wells in the northwest part of the study area. The northeast stress relieve center has large gradient with most developed fractures. However, the center, southwestern and southeast stress release centers (blue area) have small gradient with developed tectonic fractures.

The area with low stress value (blue) is the stress releasing area. In the difference stress contour map (Figure 6(c), $-3000 \mathrm{~m}$ ), the difference stress values are lower in the northeast, center and southwest area than these of other regions. The lowest difference stress values are mainly concentrated in the JY1 wells in the north and northwest part of the study area. The northeast stress relieve center has large gradient with most developed fractures. However, the center and southwestern stress release centers (blue area) have small gradient with developed tectonic fractures. 

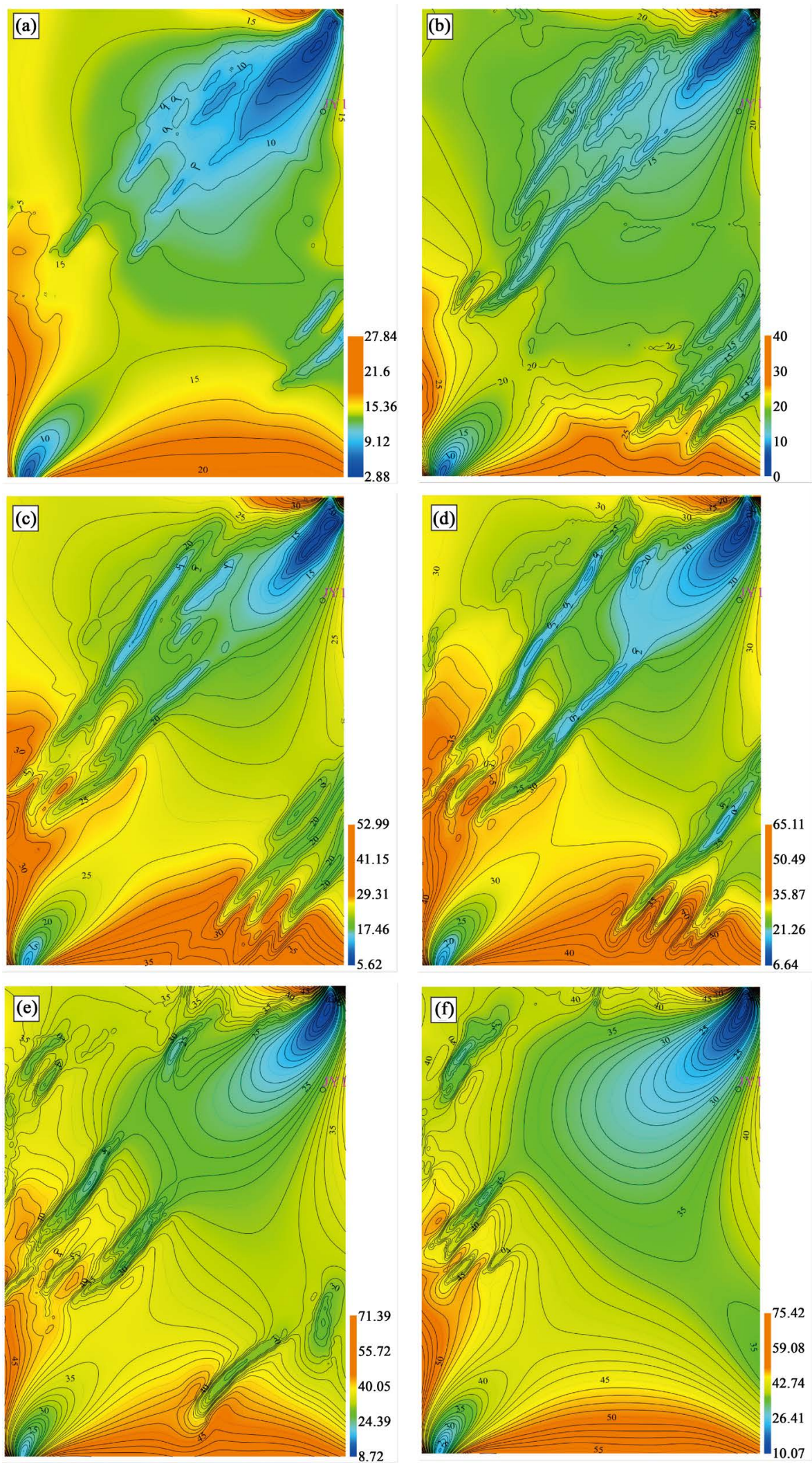

Figure 6. The difference stress contour map of different heights in the Jiaoshiba area. (a) height $-1800 \mathrm{~m}$; (b) height -2400 m; (c) height $-3000 \mathrm{~m}$; (d) height $-3600 \mathrm{~m}$; (e) height $-4200 \mathrm{~m}$; (f) height $-4800 \mathrm{~m}$. 
The area with low stress value (blue) is the stress releasing area. In the difference stress contour map (Figure 6(d), $-3600 \mathrm{~m}$ ), the difference stress values are lower in the northeast, center, southeast and southwest area than these of other regions. The lowest difference stress values are mainly concentrated in the JY1 wells in the north part of the study area. The northeast stress relieve center has large gradient with most developed fractures. However, the center, southwestern and southeast stress release centers (blue area) have small gradient with developed tectonic fractures.

The area with low stress value (blue) is the stress releasing area. In the difference stress contour map (Figure $6(\mathrm{e}),-4200 \mathrm{~m})$, the difference stress values are lower in the northeast, west, southeast and southwest area than these of other regions. The lowest difference stress values are mainly concentrated in the JY1 wells in the north part of the study area. The northeast stress relieve center has large gradient with most developed fractures. However, the west, southwestern and southeast stress release centers (blue area) have small gradient with developed tectonic fractures.

The area with low stress value (blue) is the stress releasing area. In the difference stress contour map (Figure 6(f), $-4800 \mathrm{~m}$ ), the difference stress values are lower in the northeast, northwest and southwest area than these of other regions. The lowest difference stress values are mainly concentrated in the JY1 wells in the north and west part of the study area with a large area favoring the fracture developing. The northeast stress relieve center has large gradient with most developed fractures. However, the northwest and southwest stress release centers (blue area) have small gradient with developed tectonic fractures.

\section{Conclusions and Discussion}

The contour map of difference stress values by the simulation of the present tectonic stress field suggests that there is a large difference with the development of fractures in different depths. However, the areas in favor of the development of fractures are stripped and mostly distributed in the northeast, middle and southwest of Jiaoshiba area. Several areas are in the south east of the study area. These areas are close to the distribution of faults.

Unfortunately, the wells with hydraulic fracturing data in the Jiaoshiba area are not enough to complete the verification of simulation of the tectonic differential stress values. In addition, the regional stress field is so large by the elastic plastic incremental method in this study, and the data of sedimentary facies are relatively inaccurate, which affect the division of the type of media and the degree of sedimentary microfacies. Therefore, it is necessary to carry out the further study of the simulation and the prediction of fracture zone.

\section{References}

[1] Zou, C.N., Zhu, R.K., Wu, S.T., et al. (2012) Types, Characteristics, Genesis and Prospects of Conventional and Unconventional Hydrocarbon Accumulations: Taking Tight Oil and Tight Gas in China as an Instance. Acta Petrolei Sinica, 33, 173-187.

[2] Nie, H.K., Zhang, J.C. and Li, Y.X. (2011) Accumulation Conditions of the Lower Cambrian Shale Gas in the Sichuan Basin and Its Periphery. Acta Petrolei Sinica, 32, 959-967.

[3] Chen, S.B., Zhu, Y.M., Wang, H.Y., et al. (2011) Characteristics and Significance of Mineral Compositions of Lower Silurian Longmaxi Formation Shale Gas Reservoir in the Southern Margin of Sichuan Basin. Acta Petrolei Sinica, 32, 775-782.

[4] Guo, T.L. and Zhang, H.R. (2014) Formation and Enrichment Mode of Jiaoshiba Shale Gas Field, Sichuan Basin. Petroleum Exploration and Development, 41, 28-36. http://dx.doi.org/10.1016/S1876-3804(14)60003-3

[5] Anderson, C.A. and Bridwell, R.J. (1980) A Finite Element Method for Studying the Transient Non-Linear Thermal Creep of Geological Structures. Methods Geomechanic, 4, 255-276. http://dx.doi.org/10.1002/nag.1610040305

[6] Zhang, H.F. and Zhang, W.X. (1989) Petroleum Geology. Petroleum Industry Press, Beijing, 247-248.

[7] Zeng, L.B. (1998) Tectonic Stress Field and Development of Low Permeanbility Oil Field. Oil Exploration and Development, 25, 91-93.

[8] Wang, S.X., Song, H.Z. and Liu, J. (1999) Numerical Simulation of Tectonic Stress Field in Tarim Basin and Its Significance in Oil and Gas Concentration. Seismology and Geology, 21, 268-273.

[9] Zhang, J.H. and Sun, Z.S. (2001) Stress, Testing Technology of Fissile and Their Application in Oil Exploration and Exploitation. Petroleum Industry Press, Beijing, 3-25.

[10] Li, S.E., Zhang, S.H., Yue, K., et al. (2001) Tectonic Stress Field Numerical Simulation Analysis and Its Application. Petroleum Geology and Recovery Efficiency, 8, 38-40. 
[11] Wan, T.F. (1988) Paleo-Tectonic Stress Field. Geological Publishing House, Beijing, 82-87, 124-144.

[12] Wan, T.F. (1995) The Progress of Researches on Tectonic Stress Field. Earth Science Frontiers, 2, 226-235.

[13] Chen, Z.D., Meng, Q.A., Wan, T.F., et al. (2002) Numerical Simulation of Tectonic Stress Field in Gulong Depression in Songliao Basin Using Elastic-Plastic Increment Method. Earth Science Frontiers, 9, 483-492.

[14] Zhang, S.R., Wan, T.F. and Chen, J.P. (2004) Tectonic Stress Field Modeling and Fracture Prediction in T3x2-4 Strata in Xiaoquan-Xinchang Area, Western Sichuan Depression. Oil \&Gas Geology, 25, 70-80.

[15] Wei, C.G., Lei, M.S., Wan, T.F. and Jie, W.Q. (2006) Numerical Simulation of Palaeotectonic Stress Field of Yingcheng Fm in Gulong-Xujiaweizi Area: Prediction and Comparative Study of Tectoclase Development Area. Oil \& Gas Geology, 27, 78-105.

[16] Liang, H.H., Hou, J.J., Liu, S.W., et al. (1999) Tectonic Stress Field and Large Earthquake Recurrence Period in China. Seismology and Geology, 21, 51-57.

[17] Luo, Z.N., Hu, R.H. and Li, C.Q. (1996) Dynamic Parameters and Stress Field of the Beibuwan Earthquakes. South China Journal of Seismology, 16, 72-76.

[18] Huang, Y.R., Xu, Z.H., Gao, A.J., et al. (1994) The Tectonic Stress Field of Zhongyuan Oilfield by Studying Borehole Avalanche. Journal of Earthquake, 16, 195-203.

[19] Wan, T.F. (2000) Preliminary Study of Complexity of the Tectonic Stress-Strain System. Earth Science Frontiers, 7, 161-168.

[20] Huang, F.L., Luo, C.C., He, X.H., et al. (1996) Structural Trap Series in South Songliao Basin. Oil \& Gas Geology, 17, 333-336. 